\title{
RESEARCH HIGHLIGHT LKB1 restrains dendritic cell function
}

\author{
Stefanie K. Wculek ${ }^{1}$ and David Sancho ${ }^{1}$ \\ Cell Research (2019) 29:429-431; https://doi.org/10.1038/s41422-019-0169-0
}

\begin{abstract}
Three independent recent studies support the notion that liver kinase B1 (LKB1), a key nutrient sensor, controls dendritic cell (DC) function. Selective loss of LKB1 in DCs leads to their increased ability to prime effector $T$ cell responses, but the prevailing effect is the expansion of thymus-derived natural regulatory $T$ cells, creating a dominant immunosuppressive environment.
\end{abstract}

Tight regulation of T cell priming by dendritic cells (DCs) is key to maintain tissue homeostasis and orchestrate immunity. As immune sentinels, DCs control the activation of different flavors of immunity including effector $\mathrm{CD}^{+}{ }^{+}$cells and $\mathrm{CD} 4^{+}$helper $\mathrm{T}$ (Th) cells, the latter comprising IFNy-producing Th1, IL-4-producing Th2, IL-17-producing Th17 cells and follicular helper T cells (Tfh) that promote B cell differentiation in germinal centers. ${ }^{1,2}$ However, DCs also contribute to homeostasis and self-tolerance through the induction of regulatory $\mathrm{T}$ cells (Tregs), which can be Helios ${ }^{+}$ thymus-derived/natural (tTregs) or generated upon antigen exposure in the periphery (Helios ${ }^{-}$pTregs). ${ }^{1,3}$

DCs integrate environmental cues such as pathogen- or dangerassociated molecular patterns and cytokines to activate different intracellular signaling pathways. Those adaptions lead to adjustment of antigen uptake, processing and presentation on $\mathrm{MHC}$ molecules (signal 1), expression of co-stimulatory molecules (signal 2) and production of specific cytokines (signal 3) by DCs to modulate induction of effector and regulatory $T$ cell responses. Different DC subsets include plasmacytoid (pDCs), conventional type 1 (cDC1s) and 2 (cDC2s) DCs, and express a varying repertoire of pattern recognition receptors sensing those extracellular cues culminating in diverse functional features.

Metabolic alterations in DCs upon sensing the environment have emerged as an essential mechanism for control of DC function in the regulation of adaptive immune responses. ${ }^{2,4}$ Resting or tolerogenic DCs preferentially display an active catabolic energy metabolism via the Krebs cycle and oxidative phosphorylation (OXPHOS) that can be fueled by fatty acid oxidation. Immunogenic activation of DCs generally fosters an anabolic metabolism characterized by enhanced glycolysis, and fatty acid synthesis to drive the extension of the Golgi apparatus and endoplasmic reticulum for cytokine production. ${ }^{4}$ The early induction of glycolysis regulates many aspects of immunogenic DC activation, including migration, upregulation of MHC and co-stimulatory molecules, and T cell stimulation. ${ }^{2}$ The metabolic adaption of DCs upon stimulation is, at least partially, regulated by a balance of AKT/mTOR/HIF and AMPK signaling pathways. ${ }^{2,4}$ Activation of mTOR can sustain immunogenic DC activation, while active AMPK is associated with resting and tolerized DCs. However, the precise pathways regulating the metabolic state that drive tolerogenic DC function are poorly understood. ${ }^{2,4}$
LKB1 is a serine/threonine kinase that can activate AMPK upon low intracellular ATP to induce catabolic oxidative metabolism. As such, LKB1 is implicated in modulating metabolism, survival, differentiation and functional features of hematopoietic stem cells, effector $\mathrm{CD}^{+}$and $\mathrm{CD}^{+} \mathrm{T}$ cells as well as Tregs in AMPKdependent and -independent fashions. ${ }^{5-7}$ Also, LKB1 is phosphorylated on Ser428 in lipopolysaccharide (LPS)-stimulated macrophages and restricts their pro-inflammatory functions by inhibition of NF-KB signaling, likely via binding to IKB kinase (IKK) $\beta .^{8}$

To dissect the contribution of LKB1 to DC function, three independent studies have analyzed CD11c-Cre LKB1 ${ }^{\mathrm{f} / \mathrm{f}}(\mathrm{CD} 11 \mathrm{c} \Delta \mathrm{LKB} 1)$ mice (Fig. 1). ${ }^{9-11}$ They find an enlarged tTreg pool throughout the body of $C D 11 c \triangle L K B 1$ mice, including the thymus, which protects them from experimental allergy or autoimmunity but makes them more susceptible to grafted tumors. LKB1 is phosphorylated in intratumoral DCs, ${ }^{11}$ while Escherichia coli or LPS stimulation downregulates LKB1 expression in DCs, which associates with expansion of tTregs. ${ }^{9}$ Two studies show that Tregs of CD11c $\Delta$ LKB1 mice express higher levels of immune-suppressive molecules and display an enhanced suppressive activity limiting $T$ cell proliferation. ${ }^{10,11}$ Further, Wang et al. ${ }^{11}$ demonstrate that LKB1-deficient DCs express higher levels of Treg-inducing IL-2, indoleamine 2, 3-dioxygenase (IDO) 1, arginase (Arg) 2 and integrin beta (Itgb) 8 than wild-type DCs. These factors contribute to induction of mTOR signaling in tTregs and enhance tTreg proliferation.

Notably, LKB1-deficient splenic DCs (subsets) display enhanced MHC and co-stimulatory molecule expression, foremost OX40-ligand (OX40L) and CD86, the latter also being elevated on CD11c $\Delta$ LKB1 DCs in the thymus. ${ }^{9-11}$ Indeed, Pelgrom et al. identify the thymic $\mathrm{CD} 11 \mathrm{~b}^{+} \mathrm{cDC2}$ subset, which is associated with regulation of tTreg responses, ${ }^{12}$ but not thymic $\mathrm{CDC} 1 \mathrm{~s}$ or $\mathrm{pDCs}$, to be a key player in inducing tTregs upon LKB1 loss. Mechanistically, high CD86 expression, driven by enhanced phospholipase C- $\beta 1$ (PLC- $\beta 1$ ) expression and calcium signaling in thymic CD11c $\Delta$ LKB1 $C D C 2 s$, potentiates tTreg induction. ${ }^{10}$ Frequencies of $C D C 2 s$ are also increased in thymi of $C D 11 \mathrm{C} \triangle \mathrm{LKB} 1 \mathrm{mice}$, likely further fostering induction of tTregs. ${ }^{10,11}$ Moreover, thymic CD11c $\Delta$ LKB1 $\mathrm{CDC} 2 \mathrm{~s}$ express higher levels of CCR7, ${ }^{10}$ in line with increased presence and CCR7 expression of migratory DCs in lymph nodes and augmented DC-Treg interaction. ${ }^{10,11}$ Peripheral CD11c $\triangle$ LKB1 CDC2s, but not $\mathrm{CDC1s}$, also induce additional tTreg proliferation outside the thymus. ${ }^{10}$ Chen et al. ${ }^{9}$ report an additional contributing mechanism by showing that LKB1 loss in splenic or lymph node DCs induces non-canonical NF-KB (p65) activation and subsequent upregulation of OX40L, which engages OX40 that is highly expressed on Tregs mediating their expansion in the periphery.

Interestingly, the increased $T$ cell-priming ability of LKB1deficient DCs is not restricted to tTregs. LPS- and ovalbuminstimulated CD11c $\mathrm{LKB} 1 \mathrm{DCs}(\mathrm{GM}-\mathrm{DCs})$ generated in vitro more

"Immunobiology laboratory, Fundación Centro Nacional de Investigaciones Cardiovasculares "Carlos III" (CNIC), Madrid, Spain

Correspondence: David Sancho (dsancho@cnic.es)

Published online: 25 April 2019 


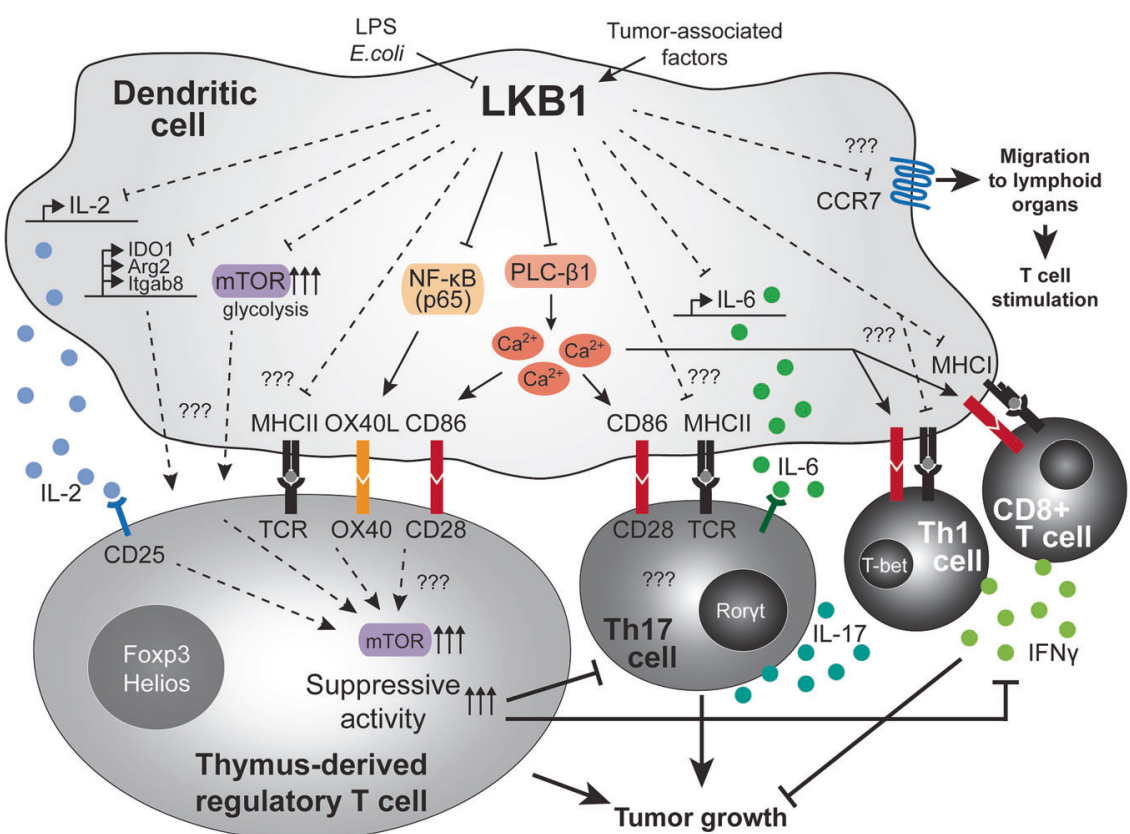

Fig. 1 LKB1 signaling in dendritic cells limits their T cell-activating potential. LKB1 is phosphorylated in DCs in the tumor microenvironment, while it is depleted by LPS or E. coli favoring Treg expansion. LKB1 limits the ability of DCs to induce T cell priming by repressing a variety of activating pathways. These effects lead to LKB1-deficient DCs to promote dysregulated T cell effector activity, with predominant increase in thymus-derived regulatory T cell priming but also increased priming of pro-immunogenic effector Th17, Th1 and CD8 ${ }^{+} \mathrm{T}$ cells. Mechanistically, upon loss of LKB1, DCs enhance their expression of MHC molecules, co-stimulatory molecules (e.g., CD86, OX40L), cytokines (e.g., IL-6, IL-2) and migration receptors (e.g., CCR7) - all of which contribute to enhanced T cell priming. The predominant activation of regulatory $T$ cells and Th17 cells upon LKB1 deletion in DCs contributes to tumor growth

profoundly induce IFNY- and/or IL-17-producing effector CD8 ${ }^{+}$ and $\mathrm{CD}^{+} \mathrm{T}$ cells after transfer into wild-type mice, which may be related to their enhanced migration to draining lymph nodes and co-stimulatory molecule expression. ${ }^{10}$ The effects of those immunogenic roles of LKB1 loss in DCs are likely dampened in vivo by Treg accumulation in CD11c $\Delta$ LKB1 mice. Nevertheless, Wang et al. observe enhanced generation of Th17 cells by LKB1deficient CD11c $\Delta$ LKB1 DCs ex vivo and in vivo, which is partially corroborated by Pelgrom et al. ${ }^{10,11}$ Elevated production of IL- 6 by CD11c $L$ LKB1 DCs may cause Th17 induction, which also contributes to the tumor susceptibility of CD11C $\Delta$ LKB1 mice. ${ }^{11}$

Finally, given the involvement of LKB1 in controlling cellular metabolism ${ }^{5-7}$ and the influence of metabolic adaptions on DC functions, ${ }^{2,4}$ the studies from Wang et al. and Pelgrom et al. analyze metabolic parameters and regulation of nutrient-sensing signaling pathways such as AMPK, mTOR and HIF1a in CD11c LLKB1 DCs, with some contrasting results. Pelgrom et al. ${ }^{10}$ report enhanced glucose uptake and unaltered or even reduced mitochondrial fitness, such as mitochondrial mass and membrane potential, in splenic (and thymic) CD11c L LKB1 CDC1s and CDC2s. Wang et al. corroborate an elevated extracellular acidification rate (ECAR) by LKB1-deficient splenic DCs, but find a significantly augmented oxygen consumption rate, a readout for OXPHOS. ${ }^{11}$ Those partially contradicting findings, which may be due to FMSlike tyrosine kinase 3 ligand (FLT3L)-mediated DC expansion, limit the potential to correlate a metabolic state or adaption of CD11c $L$ LKB1 DCs with their observed functional alteration. Nevertheless, the enhanced glycolytic activity of CD11c $L$ LKB1 DCs together with the observation of an altered cholesterol metabolism and intracellular lipid accumulation ${ }^{11}$ may associate with the metabolism of activated DCs. ${ }^{4}$

In regard to signaling pathways controlling cell metabolism, the three studies concur in pointing out that LKB1 function in DCs is independent of AMPK, the well-known LKB1 downstream target, ${ }^{5}$ by analyzing CD11c-Cre AMPKa ${ }^{f / f}$ (and AMPKa2 $2^{\mathrm{f} / \mathrm{f}}$ ) mice. ${ }^{9-11}$ Of note, Pelgrom et al. and Wang et al. find enhanced mTOR signaling in thymic and splenic LKB1-deficient DCs, respectively. Treatment of thymic $C D C 2 s$ with the mTOR inhibitor rapamycin blocks their potential to induce Treg expansion, but not the enhanced expression of CD86 on DCs. ${ }^{10}$ In line, mTOR loss in LKB1-deficient splenic DCs of CD11c-Cre LKB1 ${ }^{\mathrm{f} / \mathrm{f}} \mathrm{mTOR}^{\mathrm{f} / \mathrm{f}}$ mice has a small effect on the increased co-stimulatory molecule expression by DCs, but significantly decreases the expansion of Tregs and mTOR activation in Tregs. ${ }^{11}$ Those effects appear, however, independent from HIF1a, which was previously shown to act downstream of mTOR controlling DC metabolism, ${ }^{2,4}$ as genetic HIF1a depletion in CD11C $\Delta$ LKB1 mice does not alter DC phenotype or function. ${ }^{11}$ In contrast, Th17 induction potential or IL-6 expression of LKB1-deficient DCs is not influenced by mTOR deletion, suggesting alternative control mechanisms by LKB1. ${ }^{11}$

Overall, LKB1 emerges as a fundamental regulator of the core $D C$ function to control $T$ cell responses and maintain their immunological quiescence, at least partially via limiting DC migration, co-stimulatory molecule (CD86 and OX40L) and cytokine (IL-6) expression (Fig. 1). ${ }^{9-11}$ LKB1 loss in DCs results in their uncontrolled stimulation of $\mathrm{T}$ cells, foremost of Tregs by $\mathrm{CDC} 2 \mathrm{~s}$ in the thymus and periphery as well as peripheral Th17 cells. Prevention of mTOR signaling in DCs, likely in concert with limiting glycolytic metabolism, ${ }^{2-4}$ appears to account for aspects of LKB1-mediated regulation of T cell immunity by DCs, such as Treg homeostasis. These studies open a research avenue for the dissection of LKB1 pathway(s) regulating DC function and metabolism, which may offer potential targets to manipulate immunity and tolerance.

\section{REFERENCES}

1. Merad, M., Sathe, P., Helft, J., Miller, J. \& Mortha, A. Annu. Rev. Immunol. 31, 563-604 (2013).

2. Patente, T. A., Pelgrom, L. R. \& Everts, B. Curr. Opin. Immunol. 58, 16-23 (2019).

3. Pletinckx, K., Döhler, A., Pavlovic, V. \& Lutz, M. B. Front. Immunol. 2, 1-16 (2011).

4. O'Neill, L. A. J. \& Pearce, E. J. J. Exp. Med. 213, 15-23 (2015). 
5. Blagih, J., Krawczyk, C. M. \& Jones, R. G. Immunol. Rev. 249, 59-71 (2012).

6. He, N. et al. Proc. Natl Acad. Sci. USA 114, 12542-12547 (2017).

7. Yang, K. et al. Nature 548, 602-606 (2017).

8. Zou, M.-H. et al. J. Biol. Chem. 290, 2312-2320 (2014).
9. Chen, S. et al. Nat. Commun. 9, 5298 (2018).

10. Pelgrom, L. R. et al. Cell Res. https://doi.org/10.1038/s41422-019-0161-8 (2019)

11. Wang, Y. et al. Cell Res. https://doi.org/10.1038/s41422-019-0157-4 (2019).

12. Proietto, A. I. et al. Proc. Natl Acad. Sci. USA 105, 19869-19874 (2008). 